Author's version of

\title{
Sample size effects on grain boundary sliding
}

\author{
Jicheng Gong, and Angus J. Wilkinson
}

Department of Materials, University of Oxford, Oxford, OX1 3PH, UK

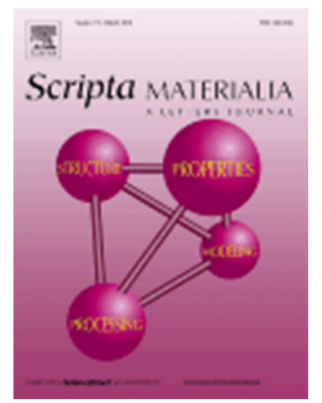

Copy of record is published as

Scripta Materialia, (2016) Volume 114, 17-20

The paper is available at:

http://dx.doi.org/10.1016/i.scriptamat.2015.11.029 


\title{
Sample Size Effects on Grain Boundary Sliding
}

\author{
Jicheng Gong $^{\mathrm{a}}$ and Angus J. Wilkinson ${ }^{\mathrm{a}^{*}}$ \\ ${ }^{a}$ Department of Materials, University of Oxford, Parks Road, \\ Oxford, OX1 3PH, United Kingdom
}

\begin{abstract}
Grain boundary sliding is an important deformation mechanism that contributes to creep and superplastic forming. In tin-based lead-free solders grain boundary sliding can make significant contributions to in service performance. Novel microcompression tests designed to isolate individual grain boundaries and assess their mechanical resistance to sliding were conducted on tin. The boundary sliding deformation was more obvious for smaller sample cross-sections and made a larger contribution to the overall deformation. As with dislocation and twinning mediated plasticity there was a significant size effect in which the resistance to grain boundary sliding increases as the sample size is reduced.
\end{abstract}

Keywords: grain boundary sliding; tin; micromechanics

*Corresponding author. Email: angus.wilkinson@materials.ox.ac.uk 


\section{Introduction}

Grain boundary sliding can be a damaging processes when it occurs during service loading but is also of great advantage when employed in superplastic forming in metals and ceramics [1-5]. It is well established that grain boundary sliding requires elevated homologous temperature and a relatively fine grain size. Tin-based alloys mostly based on silver and copper additions are the major group of materials developed as replacements for lead-tin eutectic based solders which are being replaced as a result of environmental concerns. At room temperature tin is already at $\sim 55 \%$ of its relatively low melting point (505 K) and of course operating temperatures are even higher. $\beta$-Sn makes up the largest volume fraction in the majority of lead free solder alloys and grain boundary sliding has been identified as a significant deformation mechanism $[6,7]$ especially when the grain size is small. Thermal cycling experienced by ball grid arrays in-service can lead to considerable changes within the initial microstructure leading to very inhomogeneous deformation and failure linked to strain localisation in regions where grain boundary sliding operates.

Significant grain size effect on the creep rate in grain boundary sliding and superplastic deformation of fine grained polycrystals is a well-known phenomenological observation with grain size exponents of -2 to -3 reported in metals and ceramics $[2,4,5]$. Observations on polycrystal ensembles prevent a clear separation of local driving force at a particular grain boundary and its resistance to sliding. The constraints of the three dimensional grain boundary network are prohibitively complex to unravel. However the observations of cooperative movement of groups of grains $[4,8]$ gives a clear indication that different grain boundary types have strongly differing resistance to grain boundary sliding. Work on grain boundary engineered $\mathrm{Ni}$ has shown that altering the geometrical nature of boundary types can dramatically enhance the overall resistance to creep and grain boundary sliding [9], while chemical doping of geometrically similar grain boundaries in ceramics has been shown to 
affect their propensity for boundary sliding [1]. The importance of grain boundary sliding in the deformation and failure of solder joints motivates this fundamental study of sliding at individual grain boundaries in $\beta$-Sn. Here we demonstrate the possibilities of using a focused ion beam to isolate a grain boundary segment within a small volume (few micron to submicron) which can be tested in compression (and shearing) using a nanoindenter.

Since the seminal work of Uchic et al [10] there has been a huge growth in the use of mechanical testing at the micron and sub-micron length scales. The vast majority of the work has used compression pillar geometries [10-22], though work on cantilevers in bending [2328], and tensile testing [29, 30] at these length scales has been reported. Dislocation mediated plasticity in single crystal test pieces has been the most highly studied process for which face centred [10,13,14, 17-19, 22, 28-32], body centred cubic $[11,12,16]$, and hexagonal close packed metals [26, 27] have been investigated. Deformation twinning has also received some attention in two hexagonal close packed metals $\mathrm{Ti}$ and $\mathrm{Mg}$ [20, 21]. For dislocation mediated plasticity strong size effects are evident with the flow stress values increasing as test diameters $d$ get smaller with exponents of approximately $-2 / 3$ often reported for the face centred cubic metals [13] while the exponent is -1 for both FCC and HCP in bending [27]. The stress required for the onset of deformation twinning has also been shown to be size dependent with a size exponent of -1 being reported in Ti [21]. Grain boundary sliding has not previously been tested using such small material volumes but length scale effects are to be expected.

\section{Methods}

Square cross section samples were prepared by focused ion beam (FIB) for compression testing using a nanoindenter with a flat diamond tip. The raw material was a high purity (99.99\%) tin bar supplied by Goodfellow. The bar was cut into small blocks 
$(5 \mathrm{~mm} \times 5 \mathrm{~mm} \times 10 \mathrm{~mm})$, which were then mounted in resin. These pure tin samples were heated in the resin mounts to the molten state, and then slowly cooled down to the solid state to remove the effect of pre-deformation and cutting damage. Directional solidification was implemented to control the alignment of grain boundaries inside the sample blocks. After solidification, the sample surface was carefully polished using colloidal Silica. Electron backscatter diffraction (EBSD) was used to map the crystal orientation and grain boundaries. Rectangular shaped micro-pillars with accurate controlling of the dimensions and the position of a boundary inside a pillar were machined on the selected grain boundary using a Zeiss Nvision 40 FIB-SEM system. The boundary was selected on the basis that it ran at $\sim 45^{\circ}$ to the sample surface normal which would become the compressive axis in the test pillars and so would have a large shear stress resolved upon it to promote grain boundary sliding. Micro-pillars of various dimensions were cut in each of the two crystals and spanning the grain boundary. The micro-pillar compression tests were conducted on a NanoXP nanoindentation platform equipped with a flat diamond punch which had been produced by FIB machining the end of a blunted Berkovich indenter tip. Although the indenter system is inherently load controlled the tests were conducted with the feedback system set to maintain a constant displacement rate during the tests. A target displacement rate of $20 \mathrm{~nm} / \mathrm{s}$ was used for all but one slower rate test $(2 \mathrm{~nm} / \mathrm{s})$ on a $2 \mu \mathrm{m}$ wide bi-crystal pillar. A separate temperature control system was implemented to ensure all the tests were conducted at $20^{\circ} \mathrm{C}$. The pillars were imaged using the SEM column of a Zeiss Nvision 40 FIB-SEM instrument.

\section{Results and Discussion}

The test pieces had the selected grain boundary oriented at $\sim 45^{\circ}$ to the load axis and multiple test pieces of sizes from $5 \mu \mathrm{m}, 2 \mu \mathrm{m}, 1 \mu \mathrm{m}$, and $500 \mathrm{~nm}$ where prepared from the same grain boundary. The crystal orientations determined using EBSD are illustrated in pole figures 
(figure 1) which show that the c-axis was close (within $82^{\circ}$ ) to perpendicular to the load axis for crystal 1 and somewhat further away from this (within $73^{\circ}$ ) for crystal 2 . The two crystals have a-axes that are within $18^{\circ}$ and $26^{\circ}$ of the load axis and these a-axes are only $8^{\circ}$ apart. The rotation between the two crystals can be described approximately by a $65^{\circ}$ rotation about an axis close to the almost shared [100] axis.

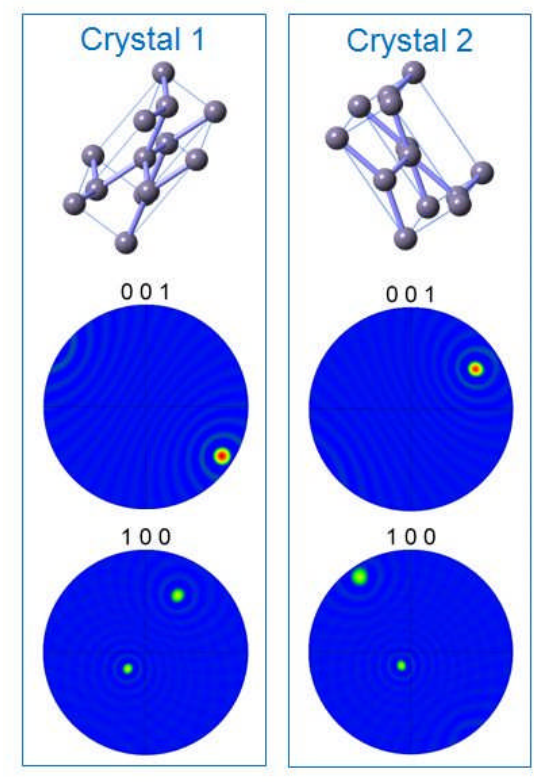

Figure 1: Orientations of the two body-centred-tetragonal $\beta$-tin crystals. The unit cells (top) are shown as viewed along the compression axis which is at the centre of the pole figures (bottom) calculated from EBSD measurements and presented in stereographic projection.

Micro-pillars of similar sizes were also cut from each of the two grains making up the bicrystal pillars. Figure 2 ( $a$ and $b$ ) shows example engineering stress-displacement curves for compression tests for different pillar sizes on both single crystals and bi-crystal pillars. We prefer to use displacement rather than strain as deformation at the base of the pillars is not negligible and can lead to significant over estimation of the strain. The stress at the onset of major displacement events were obtained from such curves. For the smaller test piece sizes $(2 \mu \mathrm{m}, 1 \mu \mathrm{m}$, and $500 \mathrm{~nm})$ abrupt load drops were seen as a result of significant displacement bursts but these are supressed at the largest pillar size. This is true for both the single crystal 
and bi-crystal pillars. A significant difference between the single crystal and bi-crystal data can be seen in the plastic response. The single crystal pillars show a general increase in the engineering stress (ie hardening) with imposed displacement interspersed by abrupt load drops, while for the bi-crystals the flow stress tends to decrease once the initial deformation event has occurred though again occasional load drops are also seen in these tests. The gradient of the initial loading part of these stress-displacement plots is systematically somewhat smaller than for the unloading part which suggests that some plastic deformation is occurring during the initial loading. This is true for both the single crystal and bi-crystal tests. The peak loads at the onset of the initial strain bursts have been used to calculate the shear stress on the grain boundary plane and these are shown as a function of pillar size in figure $2 \mathrm{c}$ where a significant 'smaller is stronger' size effect is evident. Compressive flow stress values are also shown in figure $2 \mathrm{c}$ for the single crystal tests in the two grain orientation and for the bi-crystals. The single crystals both show significantly increased strength at lower pillar sizes and for $1 \mu \mathrm{m}$ and $0.5 \mu \mathrm{m}$ size the single crystal pillars are very much stronger than the bi-crystal pillars. For the larger sizes tested $(2 \mu \mathrm{m}$ and $5 \mu \mathrm{m})$ the bicrystal is of similar strength to the two single crystals. One $2 \mu \mathrm{m}$ bi-crystal pillar was tested at a slower displacement rate of $2 \mathrm{~nm} / \mathrm{s}$ compared to $20 \mathrm{~nm} / \mathrm{s}$ for the other tests. The stress required for this slower test was toward the bottom of the range of values obtained at the faster rate. 

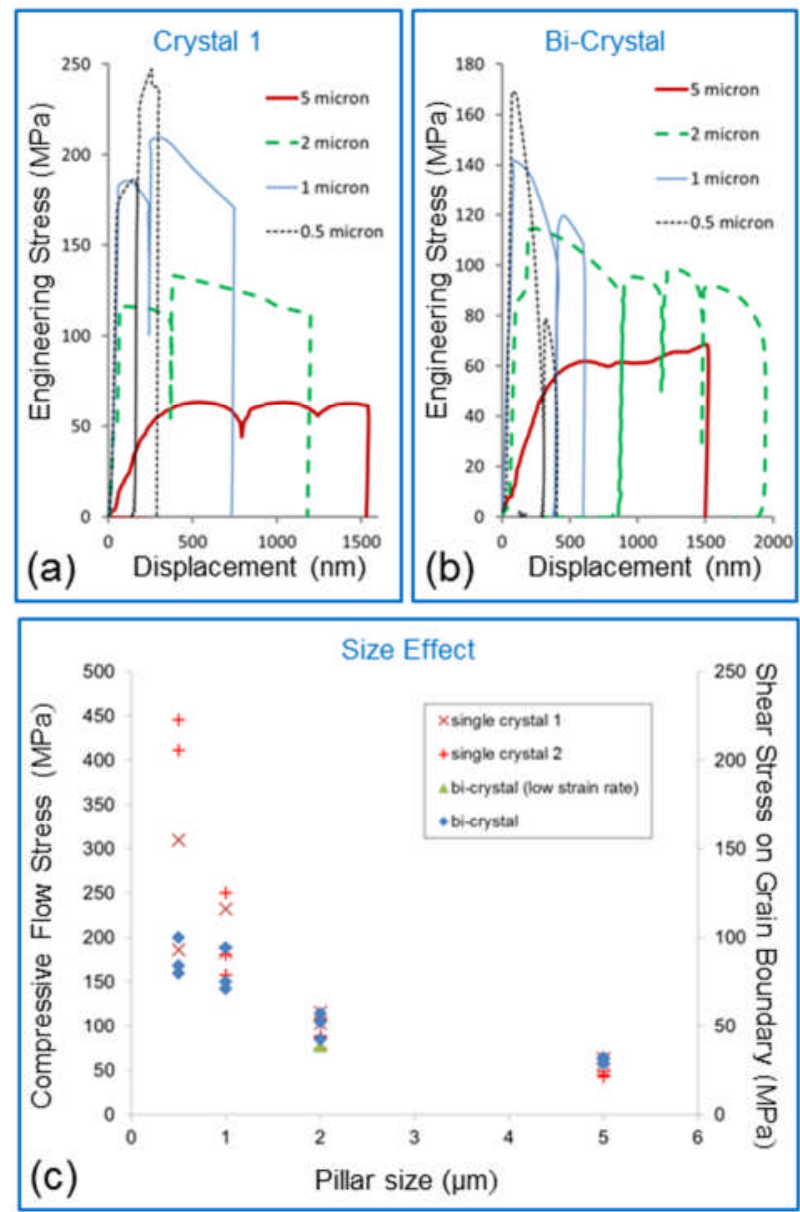

Figure 2: Micro-mechanical data from single crystal and bi-crystal compression tests. Example engineering stress versus displacement curves at different sample widths for (a) single crystal 1, and (b) bi-crystals. (c) Compressive stress (left hand axis) required for plastic deformation as a function of sample width for single crystal and bi-crystal samples. For bi-crystals shear stress on the grain boundary is also indicated (right hand axis).

Figure 3 shows example scanning electron microscope (SEM) images before and after testing of some of the bi-crystal pillars of different sizes, and for comparison some of the deformed single crystal pillars. For the bi-crystal pillars deformation is localised on the grain boundary plane and is most obvious for the smaller pillars. The highly localised shearing at the grain boundary reduces the grain boundary area during the test. This is the reason for the reduction in load and engineering stress required during deformation of these pillars. In the single crystal pillars the deformation is less localised. A large strain has been imposed by the end of 
the tests however it is clear that although one slip system dominates multiple slip has occurred. This multiple slip is evident in the increased cross-sectional area of the pillars which contributes to the increasing load and engineering stress throughout the single crystal compression tests. The large strain and multiple slip prohibits unambiguous identification of the predominant slip system, however, for the larger bi-crystal pillars there is evidence of slip on similar slip systems as for the single crystal case.

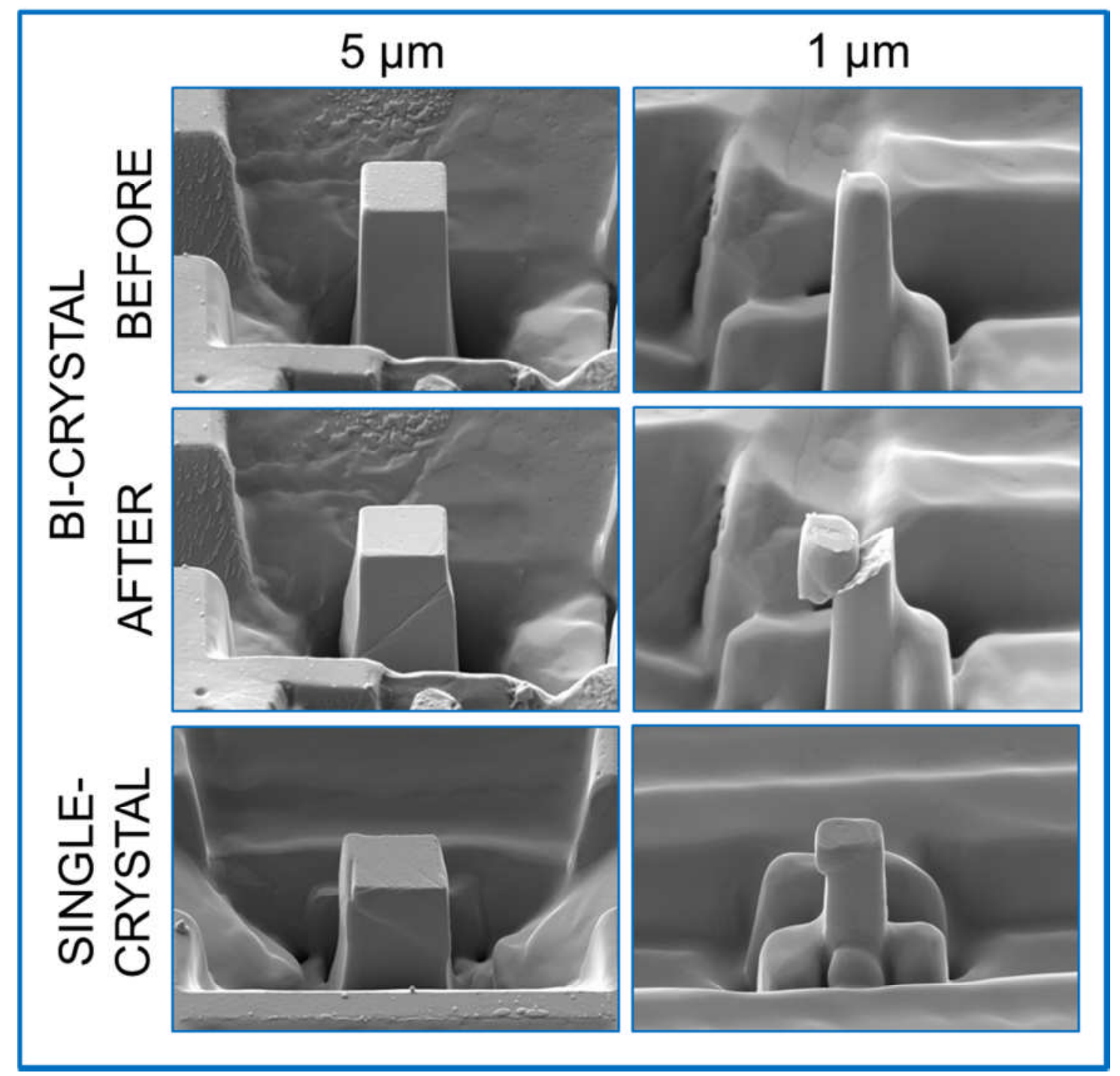

Figure 3: Example scanning electron microscope images of micro-pillars. Larger $5 \mu$ m wide pillars on left, and, smaller 1 m pillars on right. Top two rows show examples of pillars before testing (bi-crystals), while middle and bottom rows show bi-crystal and single crystal pillars after testing. 
For each bi-crystal pillar SEM images were used to measure the vertical displacement accommodated by grain boundary sliding. The displacements generated by grain boundary sliding are always smaller than the total plastic displacement recorded in the loaddisplacement data. This again suggests that further deformation modes in addition to grain boundary sliding are operating. However figure 4 shows the striking result that there is a dramatic increase in the fraction of the total plastic deformation accommodated by grain boundary sliding as the pillar size is reduced. For the larger pillars $(2 \mu \mathrm{m}$ and $5 \mu \mathrm{m})$ grain boundary sliding makes only a small contribution $(\sim 10 \%)$ to the deformation however at the smallest size $(0.5 \mu \mathrm{m})$ grain boundary sliding becomes the dominate mode and accounts for $\sim 70 \%$ of the deformation.

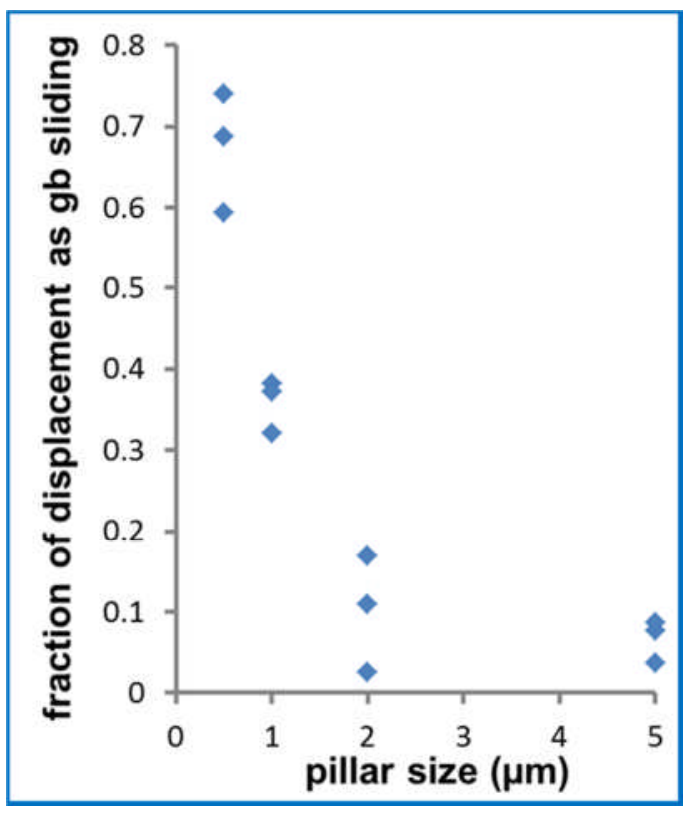

Figure 4: Fraction of deformation contributed by grain boundary sliding in bi-crystal tests as a function of pillar width. Total displacement is taken from nanoindenter data, while displacement generated by grain boundary sliding is obtained from measurement of SEM images. 
The observation of slip features and the low apparent modulus on loading suggests that there is dislocation activity within the grains of the bi-crystal before the onset of grain boundary sliding. It is thought that lattice dislocations impinging on the grain boundary cause a rearrangement of pre-existing defect content on the grain boundary plane involving the movement of glissile segments that are able to escape from the pillar causing significant plastic shearing. At the larger sizes, the stresses needed for significant dislocation slip within the two single crystals are similar to those for the bi-crystal suggesting that continued slip of lattice dislocations is required to maintain deformation. At smaller sizes a smaller number of more weakly held dislocations moving during the quasi-elastic part of the test are apparently sufficient to promote continued deformation on the grain boundary. For a given displacement vector a defect passing across the sample on the grain boundary causes significantly more strain for the smaller samples than for the larger ones. For the smaller pillars there is also less likelihood of there being a sufficiently strong obstacle on the boundary preventing passage of the grain boundary dislocation. These factors tend to promote a greater fraction of the deformation being accommodated by grain boundary sliding.

In summary, we have used FIB to cut micro-compression pillars of sizes ranging from $5 \mu \mathrm{m}$ to $0.5 \mu \mathrm{m}$ in a large grained $\beta$-Sn sample so as to contain sections of the same grain boundary inclined by $45^{\circ}$ to the loading axis. Micro-compression tests on these bi-crystal samples and similar single crystals samples cut from each of the two grains showed a significant smaller is stronger effect. However, the bi-crystal samples became easier to deform than the single crystal ones at smaller sizes and post-mortem SEM clearly showed strain to be localised at the grain boundary. Although grain boundary sliding shows a size effect it is not as strong as that for lattice dislocation mediated plasticity especially at the smaller sizes and so grain boundary sliding becomes the dominate mechanism at small pillar sizes. 


\section{Acknowledgements}

The authors gratefully acknowledge funding for this work from the Engineering and Physical Science Research Council in the United Kingdom under grant EP/G004676/1. 


\section{References:}

1. Buban JP, Matsunaga K, Chen J, Shibata N, Ching WY, Yamamoto T, et al. Grain boundary strengthening in alumina by rare earth impurities. Science. 2006;311(5758):212-5.

2. Campana C, Boyle KP, Miller RE. Grain boundary motion assisted via radiation cascades in bcc Fe. Physical Review B. 2008;78(13).

3. Gomez-Garcia D, Zapata-Solvas E, Dominguez-Rodriguez A, Kubin LP. Diffusion-driven superplasticity in ceramics: Modeling and comparison with available data. Physical Review B. 2009;80(21).

4. Van Swygenhoven H, Derlet PA. Grain-boundary sliding in nanocrystalline fcc metals. Physical Review B. 2001;64(22).

5. Wang YJ, Ishii A, Ogata S. Transition of creep mechanism in nanocrystalline metals. Physical Review B. 2011;84(22).

6. Henderson DW, Woods JJ, Gosselin TA, Bartelo J, King DE, Korhonen TM, et al. The microstructure of $\mathrm{Sn}$ in near-eutectic $\mathrm{Sn}-\mathrm{Ag}-\mathrm{Cu}$ alloy solder joints and its role in thermomechanical fatigue. J Mater Res. 2004;19(6):1608-12.

7. Telang AU, Bieler TR, Choi S, Subramanian KN. Orientation imaging studies of Sn-based electronic solder joints. J Mater Res. 2002;17(9):2294-306.

8. Zelin MG, Mukherjee AK. COOPERATIVE PHENOMENA AT GRAIN-BOUNDARIES DURING SUPERPLASTIC FLOW. Acta Metall Mater. 1995;43(6):2359-72.

9. Lehockey EM, Palumbo G. On the creep behaviour of grain boundary engineered nickel. Materials Science and Engineering a-Structural Materials Properties Microstructure and Processing. 1997;237(2):168-72.

10. Uchic M, Dimiduk D, Florando J, Nix W. Sample Dimensions Influence Strength and Crystal Plasticity. Science. 2004;305:986-9.

11. Bei H, Shim S, George EP, Miller MK, Herbert EG, Pharr GM. Compressive strengths of molybdenum alloy micro-pillars prepared using a new technique. Scripta Materialia. 2007;57(5):397400 .

12. Bei H, Shim S, Pharr GM, George EP. Effects of pre-strain on the compressive stress-strain response of Mo-alloy single-crystal micropillars. Acta Materialia. 2008;56(17):4762-70.

13. Dou R, Derby B. A universal scaling law for the strength of metal micropillars and nanowires. Scripta Materialia. 2009;61:524-7.

14. Greer JR, Oliver WC, Nix WD. Size dependence of mechanical properties of gold at the micron scale in the absence of strain gradients. Acta Materialia. 2005;53(6):1821-30.

15. Jang D, Greer JR. Transition from a strong-yet-brittle to a stronger-and-ductile state by size reduction of metallic glasses. Nature Materials. 2010;9(3):215-9.

16. Kaufmann D, Moenig R, Volkert CA, Kraft O. Size dependent mechanical behaviour of tantalum. International Journal of Plasticity. 2011;27(3):470-8.

17. Kiener D, Hosemann $P$, Maloy SA, Minor AM. In situ nanocompression testing of irradiated copper. Nature Materials. 2011;10(8):608-13.

18. Kunz A, Pathak S, Greer JR. Size effects in Al nanopillars: Single crystalline vs. bicrystalline. Acta Materialia. 2011;59(11):4416-24.

19. Uchic MD, Shade PA, Dimiduk DM. Plasticity of Micrometer-Scale Single Crystals in Compression. Annual Review of Materials Research. Annual Review of Materials Research. 392009. p. 361-86.

20. Ye J, Mishra RK, Sachdev AK, Minor AM. In situ TEM compression testing of Mg and Mg-0.2 wt.\% Ce single crystals. Scripta Materialia. 2011;64(3):292-5.

21. Yu Q, Shan Z-W, Li J, Huang X, Xiao L, Sun J, et al. Strong crystal size effect on deformation twinning. Nature. 2010;463(7279):335-8. 
22. Oh SH, Legros M, Kiener D, Dehm G. In situ observation of dislocation nucleation and escape in a submicrometre aluminium single crystal. Nature Materials. 2009;8(2):95-100.

23. Armstrong DEJ, Wilkinson AJ, Roberts SG. Measuring anisotropy in Young's modulus of copper using microcantilever testing. J Mater Res. 2009;24(11):3268-76.

24. Armstrong DEJ, Wilkinson AJ, Roberts SG. Micro-mechanical measurements of fracture toughness of bismuth embrittled copper grain boundaries. Philosophical Magazine Letters. 2011;91:394-400.

25. Di Maio D, Roberts SG. Measuring fracture toughness of coatings using focused-ion-beammachined microbeams. J Mater Res. 2005;20(2):299-302.

26. Gong JC, Wilkinson A. Investigation of elastic properties of single-crystal alpha-Ti using microcantilever beams. Philosophical Magazine Letters. 2010;90(7):503-12.

27. Gong JC, Wilkinson AJ. A Micro-Cantilever Investigation of Size Effect, Solid Solution Strengthening and Second Phase Strengthening for $<a>$ prism slip in Alpha-Ti. Acta Materialia. 2011;59:5970-81.

28. Kiener D, Motz C, Schoeberl T, Jenko M, Dehm G. Determination of mechanical properties of copper at the micron scale. Advanced Engineering Materials. 2006;8(11):1119-25.

29. Kiener D, Minor AM. Source Truncation and Exhaustion: Insights from Quantitative in situ TEM Tensile Testing. Nano Letters. 2011;11(9):3816-20.

30. Kirchlechner C, Kiener D, Motz C, Labat S, Vaxelaire N, Perroud O, et al. Dislocation storage in single slip-oriented Cu micro-tensile samples: new insights via X-ray microdiffraction. Philosophical Magazine. 2011;91(7-9):1256-64.

31. Minor AM, Asif SAS, Shan Z, Stach EA, Cyrankowski E, Wyrobek TJ, et al. A new view of the onset of plasticity during the nanoindentation of aluminium. Nature Materials. 2006;5(9):697-702.

32. Shan ZW, Mishra RK, Asif SAS, Warren OL, Minor AM. Mechanical annealing and sourcelimited deformation in submicrometre-diameter Ni crystals. Nature Materials. 2008;7(2):115-9. 